\title{
Front Matter: Volume 7012
}

, "Front Matter: Volume 7012," Proc. SPIE 7012, Ground-based and Airborne Telescopes II, 701201 (14 August 2008); doi: 10.1117/12.805242

SPIE Event: SPIE Astronomical Telescopes + Instrumentation, 2008, Marseille, France 


\title{
PROCEEDINGS OF SPIE
}

\section{Ground-based and Airborne Telescopes II}

\author{
Larry M. Stepp \\ Roberto Gilmozzi \\ Editors
}

\section{3-28 June 2008 \\ Marseille, France}

Sponsored by

SPIE

SPIE Europe

Cooperating Organizations

AAS-American Astronomical Society (USA) • ASJ-Astronomical Society of Japan (Japan)

AURA-Association of Universities for Research in Astronomy, Inc. (USA) • Ball Aerospace \& Technologies

Corporation (USA) • CNRS-Centre National de la Recherche Scientifique (France) • EAS—European

Astronomical Society (Switzerland) • ESO_European Southern Observatory (Germany) • IAU-International Astronomical Union (France) • INSU-Institut National des Sciences de I'Univers (France) • LAM-Laboratoire d'Astrophysique de Marseille (France) - MPE-Max-Planck-Institut für extraterrestrische Physik (Germany) NAOJ-National Astronomical Observatory of Japan (Japan) • NASA-NASA Goddard Space Flight Center (USA) - Northrop Grumman Corporation (USA) • OAMP-Observatoire Astronomique de Marseille Provence (France) • OPTICON-Optical Infrared Coordination Network (United Kingdom) • RadioNet_Advanced Radio Astronomy in Europe (United Kingdom) • Royal Astronomical Society (United Kingdom) • Science \& Technology Facilities Council (United Kingdom) • SFO_Société Française d'Optique (France) Competitiveness Cluster: POPsud-Pôle Optique \& Photonique (France) • Optitec Sud (France)

Published by

SPIE

Part One of Three Parts

Volume 7012 
The papers included in this volume were part of the technical conference cited on the cover and title page. Papers were selected and subject to review by the editors and conference program committee. Some conference presentations may not be available for publication. The papers published in these proceedings reflect the work and thoughts of the authors and are published herein as submitted. The publisher is not responsible for the validity of the information or for any outcomes resulting from reliance thereon.

Please use the following format to cite material from this book:

Author(s), "Title of Paper," in Ground-based and Airborne Telescopes II, edited by Larry M. Stepp, Roberto Gilmozzi, Proceedings of SPIE Vol. 7012 (SPIE, Bellingham, WA, 2008) Article CID Number.

ISSN 0277-786X

ISBN 9780819472229

Published by

SPIE

P.O. Box 10, Bellingham, Washington 98227-0010 USA

Telephone +1 3606763290 (Pacific Time) · Fax +1 3606471445

SPIE.org

Copyright (C 2008, Society of Photo-Optical Instrumentation Engineers

Copying of material in this book for internal or personal use, or for the internal or personal use of specific clients, beyond the fair use provisions granted by the U.S. Copyright Law is authorized by SPIE subject to payment of copying fees. The Transactional Reporting Service base fee for this volume is $\$ 18.00$ per article (or portion thereof), which should be paid directly to the Copyright Clearance Center (CCC), 222 Rosewood Drive, Danvers, MA 01923. Payment may also be made electronically through CCC Online at copyright.com. Other copying for republication, resale, advertising or promotion, or any form of systematic or multiple reproduction of any material in this book is prohibited except with permission in writing from the publisher. The CCC fee code is $0277-786 \times / 08 / \$ 18.00$.

Printed in the United States of America.

Publication of record for individual papers is online in the SPIE Digital Library.

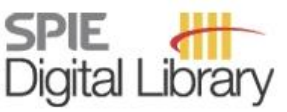

SPIEDigitallibrary.org

Paper Numbering: Proceedings of SPIE follow an e-First publication model, with papers published first online and then in print and on CD-ROM. Papers are published as they are submitted and meet publication criteria. A unique, consistent, permanent citation identifier (CID) number is assigned to each article at the time of the first publication. Utilization of CIDs allows articles to be fully citable as soon they are published online, and connects the same identifier to all online, print, and electronic versions of the publication. SPIE uses a six-digit CID article numbering system in which:

- The first four digits correspond to the SPIE volume number.

- The last two digits indicate publication order within the volume using a Base 36 numbering system employing both numerals and letters. These two-number sets start with 00, 01, 02, 03, 04, 05 , $06,07,08,09,0 A, 0 B \ldots 0 Z$, followed by $10-12,20-2 Z$, etc.

The CID number appears on each page of the manuscript. The complete citation is used on the first page, and an abbreviated version on subsequent pages. Numbers in the index correspond to the last two digits of the six-digit CID number. 


\title{
Contents
}

\section{Part One}

\author{
xxv Conference Committee \\ xxix Introduction \\ xxxi High redshift galaxy surveys (Plenary Paper) [7016-500] \\ M. Iye, National Astronomical Observatory of Japan (Japan)
}

\section{SESSION 1 PROJECT REVIEWS}

701202 The GTC project: under commissioning (Invited Paper) [7012-01]

P. Alvarez, J. M. Rodriguez Espinosa, F. J. Castro Lopez-Tarruella, M. Pi, P. Hammersley, L. Cavaller, B. Siegel, J. Molgo, J. Pancorbo, R. Ascanio, G. Prieto, J. Calvo, Instituto de Astrofísica de Canarias (Spain)

701203 The Large Binocular Telescope (Invited Paper) [7012-02]

J. M. Hill, R. F. Green, J. H. Slagle, D. S. Ashby, G. Brusa-Zappellini, J. G. Brynnel, N. J. Cushing, J. Little, R. M. Wagner, Large Binocular Telescope Observatory, Univ. of Arizona (United States)

701204 Preparing first light of LAMOST (Invited Paper) [7012-03]

X. Cui, Nanjing Institute of Astronomical Optics \& Technology (China)

701205 The Large Synoptic Survey Telescope: concept design overview (Invited Paper) [7012-04] V. L. Krabbendam, NOAO/LSST (United States)

\section{SESSION 2 INTEGRATION, COMMISSIONING, AND EARLY RESULTS}

701207 AMiBA first year observation [7012-93]

K. Lin, Institute of Astronomy and Astrophysics, Academia Sinica (Taiwan) and National Taiwan Univ. (Taiwan); C.-T. Li, Institute of Astronomy and Astrophysics, Academia Sinica (Taiwan); J.-H P. WU, National Taiwan Univ. (Taiwan); P. M. Koch, K. Umetsu, G.-C. Liu, H. Nishioka, Institute of Astronomy and Astrophysics, Academia Sinica (Taiwan); P. Altamirano, D. Kubo, ASIAA Hawaii Operation (United States); C.-C. Han, Y.-D. Huang, P. Raffin, Institute of Astronomy and Astrophysics, Academia Sinica (Taiwan); M. Kesteven, Australian Telescope National Facility (Australia); C.-W. Huang, Y.-W. Liao, F.-C. Wang, National Taiwan Univ. (Taiwan); S.-W. Chang, C.-H. Chang, Institute of Astronomy and Astrophysics, Academia Sinica (Taiwan); P. Oshiro, ASIAA Hawaii Operation (United States); S.-H. Chang, H. Jiang, M.-T. Chen, Y.-J. Hwang, Institute of Astronomy and Astrophysics, Academia Sinica (Taiwan); W. Wilson, Australian Telescope National Facility (Australia); K.-J. Chen, F. Ibanez-Romano, P. T.-P. Ho, Institute of Astronomy and Astrophysics, Academia Sinica (Taiwan); W.-Y. P. Hwang, National Taiwan Univ. (Taiwan) 
701208 New achievements of ASTE: the Atacama Submillimeter Telescope Experiment [7012-07] H. Ezawa, National Astronomical Observatory of Japan (Japan) and Nobeyama Radio Observatory (Japan); K. Kohno, Institute of Astronomy, The Univ. of Tokyo (Japan); R. Kawabe, Nobeyama Radio Observatory (Japan); S. Yamamoto, The Univ. of Tokyo (Japan); H. Inove, Institute of Astronomy, The Univ. of Tokyo (Japan); H. Iwashita, Nobeyama Radio Observatory (Japan); H. Matsuo, T. Okuda, National Astronomical Observatory of Japan (Japan); T. Oshima, T. Sakai, K. Tanaka, Nobeyama Radio Observatory (Japan); N. Yamaguchi, National Astronomical Observatory of Japan (Japan) and Nobeyama Radio Observatory (Japan); G. W. Wilson, M. S. Yun, Univ. of Massachusetts, Amherst (United States); I. Aretxaga, D. Hughes, Instituto Nacional de Astrofísica, Óptica y Electrónica (Mexico); J. Austermann, T. A. Perera, K. S. Scott, Univ. of Massachusetts, Amherst (United States); L. Bronfman, J. R. Cortés, Univ. de Chile (Chile)

701209 The architecture and initial results of the Large Millimeter Telescope control system [7012-08]

K. Souccar, Univ. of Massachusetts, Amherst (United States); D. Smith, MERLAB, P.C. (United States)

\section{SESSION 3 NEW FACILITIES AND APPROACHES I}

7012 OA Opto-mechanical design of the 3,6 m Optical Telescope for ARIES [7012-09]

C. Flebus, E. Gabriel, S. Lambotte, Advanced Mechanical and Optical Systems (Belgium); N. Ninane, Ctr. Spatial de Liège (Belgium); M. Piérard, F. Rausin, J-M. Schumacher, Advanced Mechanical and Optical Systems (Belgium)

7012 OB Current status of the Hobby-Eberly Telescope wide field upgrade and VIRUS [7012-10] R. D. Savage, J. A. Booth, K. Gebhardt, J. M. Good, G. J. Hill, P. J. MacQueen, M. D. Rafal, M. P. Smith, B. L. Vattiat, McDonald Observatory, The Univ. of Texas at Austin (United States)

$70120 \mathrm{C}$ Beating the cost curve and redefining the scientific telescope utility using 0.4-meter robotic cluster network [7012-96]

M. A. Dubberley, Z. A. Walker, B. J. Haldeman, Las Cumbres Observatory Global Telescope (United States)

7012 OD The eSMA: description and first results [7012-12] S. Bottinelli, Leiden Observatory, Leiden Univ. (Netherlands); K. H. Young, HarvardSmithsonian Ctr. for Astrophysics (United States); R. Chamberlin, Caltech Submillimeter Observatory (United States); R. P. J. Tilanus, Joint Astronomy Ctr. (United States) and Netherlands Organisation for Scientific Research (Netherlands); M. A. Gurwell, D. J. Wilner, Harvard-Smithsonian Ctr. for Astrophysics (United States); H. Shinnaga, H. Yoshida, Caltech Submillimeter Observatory Office (United States); P. Friberg, Joint Astronomy Ctr. (United States); H. J. van Langevelde, Joint Institute for VLBI in Europe (Netherlands) and Leiden Observatory, Leiden Univ. (Netherlands); E. F. van Dishoeck, Leiden Observatory, Leiden Univ. (Netherlands) and Max-Planck-Institut für Extraterrestrische Physik (Germany); M. R. Hogerheijde, Leiden Observatory, Leiden Univ. (Netherlands); A. M. Hughes, R. D. Christensen, Harvard-Smithsonian Ctr. for Astrophysics (United States); R. E. Hills, J. S. Richer, E. Curtis, Cavendish Lab., Univ. of Cambridge (United Kingdom)

7012 OE The conceptual design and simulation of 30m RIT [7012-13]

Z. Liu, D. Yichun, Z. Jin, X. Jun, J. Lin, Yunnan Astronomical Observatory (China) 
7012 OF Status of the Sardinia Radio Telescope project [7012-14]

G. Tofani, Arcetri Astrophysical Observatory, INAF (Italy); G. Alvito, Cagliari Astronomy Observatory, INAF (Italy); R. Ambrosini, Radio Astronomy Institute, INAF (Italy); P. Bolli, Cagliari Astronomy Observatory, INAF (Italy); C. Bortolotti, Radio Astronomy Institute, INAF (Italy); L. Bruca, ASI-Agenzia Spaziale Italiana (Italy); F. Buffa, A. Cattani, Cagliari Astronomy Observatory, INAF (Italy); G. Comoretto, Arcetri Astrophysical Observatory, INAF (Italy); A. Cremonini, Cagliari Astronomy Observatory, INAF (Italy); L. Cresci, Arcetri Astrophysical Observatory, INAF (Italy); N. D'Amico, G. L. Deiana, A. Fara, Cagliari Astronomy Observatory, INAF (Italy); L. Feretti, F. Fiocchi, Radio Astronomy Institute, INAF (Italy); E. Flamini, ASI_Agenzia Spaziale Italiana (Italy); F. Fusi Pecci, Bologna Astronomy Observatory, INAF (Italy); G. Grueff, G. Maccaferri, A. Maccaferri, F. Mantovani, S. Mariotti, Radio Astronomy Institute, INAF (Italy); C. Migoni, F. Messina, Cagliari Astronomy Observatory, INAF (Italy); J. Monari, M. Morsiani, Radio Astronomy Institute, INAF (Italy); M. Murgia, J. Musmeci, Cagliari Astronomy Observatory, INAF (Italy); M. Nanni, V. Natale, Radio Astronomy Institute, INAF (Italy); A. Navarrini, Cagliari Astronomy Observatory, INAF (Italy); M. Negusini, R. Nesti, L. Olmi, A. Orfei, A. Orlati, Radio Astronomy Institute, INAF (Italy); F. Palla, D. Panella, Arcetri Astrophysical Observatory, INAF (Italy); C. Pernechele, S. Pilloni, T. Pisanu, A. Poddighe, Cagliari Astronomy Observatory, INAF (Italy); M. Poloni, Radio Astronomy Institute, INAF (Italy); A. Poma, S. Poppi, I. Porceddu, Cagliari Astronomy Observatory, INAF (Italy); I. Prandoni, J. Roda, M. Roma, P. Sarti, A. Scalambra, F. Schillirò, Radio Astronomy Institute, INAF (Italy); A. Tarchi, G. P. Vargiu, Cagliari Astronomy Observatory, INAF (Italy); G. Zacchiroli, Radio Astronomy Institute, INAF (Italy)

$70120 \mathrm{G}$ The Infra-Red Imaging Survey (IRIS) system [7012-15]

K. W. Hodapp, Institute for Astronomy, Univ. of Hawaii (United States); R. Chini, Astronomical Institute of the Ruhr Univ. Bochum (Germany); B. Reipurth, Institute for Astronomy, Univ. of Hawaii (United States); R. Lemke, Astronomical Institute of the Ruhr Univ. Bochum (Germany); S. Jacobson, Institute for Astronomy, Univ. of Hawaii (United States); T. Chonis, Univ. of Nebraska, Lincoln (United States); K. D. Dement, Institute for Astronomy, Univ. of Hawaii (United States); K. Bischoff, Halfmann Teleskoptechnik (Germany)

$7012 \mathrm{OH} \quad$ A comparison of vibration damping methods for ground based telescopes [7012-16] E. H. Anderson, R. M. Glaese, CSA Engineering, Inc. (United States); D. Neill, National Optical Astronomy Observatory (United States)

\section{SESSION 4 SOLAR TELESCOPES}

7012 ol Advanced Technology Solar Telescope: a progress report (Invited Paper) [7012-17] J. Wagner, T. R. Rimmele, S. Keil, R. Hubbard, E. Hansen, L. Phelps, M. Warner, B. Goodrich, K. Richards, S. Hegwer, R. Kneale, J. Ditsler, National Solar Observatory (United States)

7012 0J European Solar Telescope (EST): project status [7012-18]

M. Collados, Instituto de Astrofísica de Canarias (Spain)

7012 OK Thermal characteristics of the solar telescope GREGOR [7012-19]

R. Volkmer, Kiepenheuer-Institut für Sonnenphysik (Germany)

7012 OL New telescopes for ground-based solar observations at submillimeter and mid-infrared [7012-20]

P. Kaufmann, Univ. Presbiteriana Mackenzie (Brazil) and Univ. Estadual de Campinas (Brazil); H. Levato, Complejo Astronômico El Leoncito (Argentina); M. M. Cassiano, Univ. 
Presbiteriana Mackenzie (Brazil); E. Correia, Univ. Presbiteriana Mackenzie (Brazil) and Instituto Nacional de Pesquisas Espaciais (Brazil); J. E. R. Costa, Instituto Nacional de Pesquisas Espaciais (Brazil); C. G. Giménez de Castro, Univ. Presbiteriana Mackenzie (Brazil); R. Godoy, Complejo Astronômico El Leoncito (Argentina); R. K. Kingsley, J. S. Kingsley, Univ. of Arizona, Steward Observatory (United States); A. S. Kudaka, Univ. Presbiteriana Mackenzie (Brazil); R. Marcon, Univ. Estadual de Campinas (Brazil) and Bernard Lyot Solar Observatory (Brazil); R. Martin, Composite Mirror Applications, Inc. (United States); A. Marun, Complejo Astronômico El Leoncito (Argentina); A. M. Melo, Univ. Presbiteriana Mackenzie (Brazil) and Univ. Estadual de Campinas (Brazil); P. Pereyra, Complejo Astronômico El Leoncito (Argentina); J.-P. Raulin, Univ. Presbiteriana Mackenzie (Brazil); T. Rose, RPG—Radiometer Physics (Germany); A. Silva Valio, Univ. Presbiteriana Mackenzie (Brazil); A. Walber, RPG - Radiometer Physics (Germany); P. Wallace, STFC Rutherford Appleton Lab. (United Kingdom); A. Yakubovich, Orbit Technology Group, Ltd. (Israel); M. B. Zakia, Univ. Estadual de Campinas (Brazil)

$70120 \mathrm{M}$ Cornelis Zwaan, open principle, and the future of high-resolution solar telescopes [7012-21] R. H. Hammerschlag, F. C. M. Bettonvil, Astronomical Institute, Utrecht Univ. (Netherlands); A. P. L. Jägers, G. Sliepen, Utrecht Univ. (Netherlands) and Technology Foundation STW, Utrecht (Netherlands)

SESSION 5 ALMA

7012 ON The Atacama Large Millimeter/submillimeter Array (Invited Paper) [7012-22] R. E. Hills, A. J. Beasley, ALMA (Chile)

701200 The array configuration design of the Atacama Compact Array [7012-118] K.-I. Morita, National Astronomical Observatory of Japan (Japan); M. A. Holdaway, National Radio Astronomy Observatory (United States)

7012 OP The European ALMA project: new design and technologies for innovative performances [7012-24]

G. Marchiori, F. Rampini, European Industrial Engineering (Italy); P. Lapeyre, Thales Alenia Space France (France)

$70120 Q$ Manufacturing and on-site assembly of the European ALMA production antennas: a status report [7012-25]

M. Apers, Thales Alenia Space France (France); G. Marchiori, M. Pozzobon, European Industrial Engineering (Italy); P. Emde, MT Mechatronic (Germany); P. Lapeyre, Thales Alenia Space France (France); M. Suita, Thales Alenia Space Italia (Italy); F. Rampini, European Industrial Engineering (Italy); S. Stanghellini, ESO (Germany)

7012 OR The metrology system of the European ALMA project: thermal and wind pointing error corrections [7012-26]

F. Rampini, G. Marchiori, L. Ghedin, European Industrial Engineering (Italy); R. Biasi, Microgate (Italy); S. Stanghellini, ESO (Germany)

SESSION 6 RADIO TELESCOPES AND THEIR TECHNOLOGY

7012 OS The Large Millimeter Telescope (Invited Paper) [7012-27]

F. P. Schloerb, Univ. of Massachusetts, Amherst (United States) 
7012 OT Working space analysis and optimization of the main positioning system of FAST cabin suspension [7012-122]

H. Li, R. Nan, National Astronomical Observatories (China); H. J. Kärcher, MT Mechatronics (Germany); W. Zhu, J. Sun, National Astronomical Observatories (China); M. Lazanowski, S. Kern, B. Strah, F. F. Wamba, Technische Univ. Darmstadt (Germany); C. Jin, National Astronomical Observatories (China)

\section{SESSION $7 \quad$ WIND LOADING ON TELESCOPES}

7012 OU The study on the scheme of dynamic measurement of the main active reflector for FAST [7012-29]

L. Zhu, J. Hu, National Astronomical Observatory (China); W. Li, Univ. of Science and Technology of China (China)

7012 OV Wind induced surface deformation of the Nobeyama 45-m radio telescope [7012-30] N. Ukita, National Astronomical Observatory of Japan (Japan)

7012 OW Wind induced image degradation (jitter) of the LSST telescope [7012-31]

D. R. Neill, J. Sebag, M. Warner, V. Krabbendam, NOAO/LSST (United States)

7012 OX Wind Evaluation Breadboard: mechanical design and analysis, control architecture, dynamic model, and performance simulation [7012-32]

M. Reyes García-Talavera, T. Viera, M. Núñez, Instituto de Astrofísica de Canarias (Spain); P. Zuluaga, Instituto de Astrofísica de Canarias (Spain) and Altran Technologies (Spain);

B. Ronquillo, M. Ronquillo, Altran Technologies (Spain); E. Brunetto, M. Quattri, ESO (Germany); J. Castro, Grantecan S.A. (Spain); E. Hernández, Instituto de Astrofísica de Canarias (Spain)

\section{SESSION 8 SEGMENTED MIRROR ALIGNMENT AND PHASING I}

7012 OY A conceptual design for the Thirty Meter Telescope alignment and phasing system [7012-125]

M. Troy, Jet Propulsion Lab. (United States); G. Chanan, S. Michaels, Univ. of California, Irvine (United States); R. Bartos, G. Bothwell, A. Give'on, R. Hein, Jet Propulsion Lab. (United States); M. Radin, Univ. of California, Irvine (United States); J. Roberts, Jet Propulsion Lab. (United States); J. M. Rodgers, Optical Research Associates (United States); L. M. Scherr, B.-J. Seo, Jet Propulsion Lab. (United States); D. Zimmerman, Univ. of California, Irvine (United States)

$70120 Z$ Active Phasing Experiment: preliminary results and prospects [7012-34]

F. Gonte, C. Araujo, R. Bourtembourg, R. Brast, F. Derie, P. Duhoux, C. Dupuy, C. Frank, R. Karban, R. Mazzoleni, L. Noethe, I. Surdej, N. Yaitskova, European Southern Observatory (Germany); R. Wilhelm, B. Luong, FOGALE nanotech (France); E. Pinna, Arcetri Astrophysical Observatory (Italy); S. Chueca, Instituto de Astrofísica de Canarias (Spain); A. Vigan, Lab. d'Astrophysique de Marseille (France) 
701210 Advances in edge sensors for the Thirty Meter Telescope primary mirror [7012-35] C. Shelton, Jet Propulsion Lab. (United States); T. Mast, Univ. of California, Santa Cruz (United States); G. Chanan, Univ. of California, Irvine (United States); J. Nelson, Univ. of California, Santa Cruz (United States); L. C. Roberts, Jr., M. Troy, Jet Propulsion Lab. (United States); M. J. Sirota, Thirty Meter Telescope Corp. (United States); B.-J. Seo,

D. R. MacDonald, Jet Propulsion Lab. (United States)

701211 Cornell Caltech Atacama Telescope primary mirror surface sensing and controllability [7012-36]

D. MacDonald, Jet Propulsion Lab. (United States); D. Woody, California Institute of Technology (United States); C. M. Bradford, Jet Propulsion Lab. (United States);

R. Chamberlin, California Institute of Technology (United States); M. Dragovan,

P. Goldsmith, Jet Propulsion Lab. (United States); S. Radford, California Institute of Technology (United States); T. Sebring, Cornell Univ. (United States); J. Zmuidzinas, Jet Propulsion Lab. (United States)

701212 Optical phasing of a segmented mirror with subnanometer precision: experimental results of the APE Internal Metrology [7012-37]

R. Wilhelm, B. Luong, A. Courteville, S. Estival, FOGALE nanotech (France); F. Gonté,

European Southern Observatory (Germany)

\section{SESSION 9 SEGMENTED MIRROR ALIGNMENT AND PHASING II}

701213 DIPSI: measure of the tip-tilt with a diffraction image phase sensing instrument [7012-38] S. Chueca, M. Reyes, Instituto de Astrofísica de Canarias (Spain); A. Schumacher, GTC (Spain); L. Montoya, Instituto de Astrofísica de Canarias (Spain)

701214 Phasing segmented telescopes with long-exposure phase diversity images [7012-39] S. Meimon, E. Delavaquerie, F. Cassaing, T. Fusco, L. M. Mugnier, V. Michau, Office National d'Études et de Recherches Aérospatiales (France) and Observatoire de Paris, CNRS, and Univ. Denis Diderot Paris 7 (France)

701215 Diffraction halo by a segmented telescope [7012-40]

N. Yaitskova, European Organisation for Astronomical Research in the Southern Hemisphere (Germany)

701216 Disentangling between low order telescope aberrations and segmentation errors using a Shack-Hartmann sensor [7012-41]

R. Mazzoleni, N. Yaitskova, L. Noethe, European Organisation for Astronomical Research in the Southern Hemisphere (Germany)

701217 Inductive edge sensors: an innovative solution for ELT segmented mirror alignment monitoring [7012-42]

D. Rozière, B. Luong, B. Fuchs, A. Périn, C. Néel, FOGALE nanotech (France); S. Lévèque, European Southern Observatory (Germany)

701218 Preliminary results obtained with the ZEUS phasing sensor within the APE experiment [7012-43]

I. Surdej, European Organisation for Astronomical Research in the Southern Hemisphere (Germany); B. Luong, FOGALE nanotech (France); A. Vigan, Lab. d'Astrophysique de Marseille (France); C. Araujo, R. Bourtembourg, R. Brast, P. Duhoux, C. Dupuy, C. Frank, 
F. Gonte, R. Karban, R. Mazzoleni, L. Noethe, N. Yaitskova, European Organisation for Astronomical Research in the Southern Hemisphere (Germany); K. Dohlen, Lab.

d'Astrophysique de Marseille (France)

\section{SESSION 10 FUTURE GIANT TELESCOPES I}

701219 The 42m European ELT: status (Invited Paper) [7012-44]

R. Gilmozzi, J. Spyromilio, European Southern Observatory (Germany)

$70121 \mathrm{~A}$ The status of the Thirty Meter Telescope project (Invited Paper) [7012-45]

J. Nelson, Univ. of California, Santa Cruz (United States); G. H. Sanders, TMT Project (United States)

7012 1B Progress on the GMT (Invited Paper) [7012-46]

M. Johns, Carnegie Observatories (United States)

7012 1C Preliminary design for an enclosure for the European Extremely Large Telescope [7012-47]

G. Murga, A. Bilbao, A. Vizcargüenaga, A. Fernández, A. Zarraoa, IDOM (Spain);

M. Schneermann, ESO (Germany)

\section{Part Two}

SESSION 11 FUTURE GIANT TELESCOPES II

7012 1D Enclosure design for Thirty Meter Telescope [7012-48]

N. Loewen, C. Breckenridge, Empire Dynamic Structures, Ltd. (Canada); A. Vasiljevic,

Association of Canadian Univs. for Research in Astronomy (Canada)

7012 IE Introduction to FAST: five hundred meter Aperture Spherical radio Telescope

(Invited Paper) [7012-49]

R. Nan, National Astronomical Observatories (China)

$7012 \mathrm{lF} \quad$ Design of the primary mirror segment support system for the E-ELT [7012-50]

L. Cavaller, J. Marrero, J. Castro, Grantecan S.A., (Spain); E. Morante, CESA S.A. (Spain); M. Ronquillo, MEDIA Consultores de Ingenieria (Spain); E. Hernández, Instituto de Astrofísica de Canarias (Spain)

$70121 \mathrm{G} \quad$ ELTs, AO wavelengths, and science output [7012-51]

A. Ardeberg, P. Linde, European Extremely Large Telescope, Lund Observatory (Sweden)

\section{SESSION 12 FUTURE GIANT TELESCOPES III}

7012 1H The Cornell Caltech Atacama Telescope status and technical progress (Invited Paper) [7012-52]

T. A. Sebring, Cornell Univ. (United States); S. Radford, California Institute of Technology (United States); R. Giovanelli, Cornell Univ. (United States); J. Glenn, Univ. of Colorado, Boulder (United States); D. Woody, California Institute of Technoloy (United States) 
$701211 \quad$ The Square Kilometre Array (Invited Paper) [7012-53]

R. T. Schilizzi, P. E. F. Dewdney, SKA Program Development Office (United Kingdom);

T. J. W. Lazio, Naval Research Lab. (United States)

$70121 \mathrm{~J}$ The E-ELT project: the feasibility study [7012-54]

G. Marchiori, S. De Lorenzi, A. Busatta, L. Giacomel, European Industrial Engineering (Italy)

\section{SESSION 13 TELESCOPE ALIGNMENT AND CONTROL I}

$70121 \mathrm{~K} \quad$ Alignment of the Pan-STARRS PS1 prototype telescope optics [7012-55]

J. S. Morgan, N. Kaiser, Pan-STARRS (United States)

$70121 \mathrm{~L} \quad$ Use of field aberrations in the alignment of the Large Binocular Telescope optics [7012-56] A. Rakich, J. M. Hill, C. J. Biddick, D. L. Miller, T. Leibold, Large Binocular Telescope Observatory (United States)

$70121 \mathrm{M}$ Prime focus active optics with the Large Binocular Telescope [7012-57]

J. M. Hill, Large Binocular Telescope Observatory, Univ. of Arizona (United States);

R. Ragazzoni, A. Baruffolo, Osservatorio Astronomico di Padova, INAF (Italy); C. J. Biddick,

O. P. Kuhn, Large Binocular Telescope Observatory, Univ. of Arizona (United States);

E. Diolaiti, Osservatorio Astronomico di Bologna, INAF (Italy); D. Thompson, A. Rakich, Large Binocular Telescope Observatory, Univ. of Arizona (United States)

$70121 \mathrm{~N} \quad$ Control analysis of the TMT primary segment assembly [7012-58]

P. M. Thompson, Systems Technology, Inc. (United States); D. G. MacMynowski, M. J. Sirota, TMT Observatory Corp. (United States)

$701210 \quad$ E-ELT primary mirror control system [7012-59]

M. Dimmler, T. Erm, B. Bauvir, B. Sedghi, H. Bonnet, M. Müller, A. Wallander, European

Southern Observatory (Germany)

7012 1P Analysis of the TMT mount control system [7012-60]

P. M. Thompson, Systems Technology, Inc. (United States); D. G. MacMynowski, M. J. Sirota, TMT Observatory Corp. (United States)

$70121 Q \quad$ Acceleration feedback control on an AT [7012-61]

B. Sedghi, B. Bauvir, M. Dimmler, European Southern Observatory (Germany)

\section{SESSION 14 AIRBORNE TELESCOPES}

$70121 R \quad$ The Stratospheric Observatory for Infrared Astronomy (SOFIA) (Invited Paper) [7012-62]

R. D. Gehrz, Univ. of Minnesota (United States); E. E. Becklin, Univ. Space Research Association, NASA Ames Research Ctr. (United States)

7012 is A balloon-borne stratospheric telescope for Venus observations [7012-63]

E. F. Young, M. A. Bullock, Southwest Research Institute (United States); A. Kraut, G. Orr,

K. Swartzlander, T. Wimer, E. Wong, P. Little, Harvey Mudd College (United States);

Y. Nakaya, Kogakuin Univ. (Japan); R. Mellon, Equinox Interscience, Inc. (United States);

L. Germann, Left Hand Design Corp. (United States) 
$70121 \mathrm{E} \quad$ E-ELT site characterization status [7012-64]

J. Vernin, UMR6525 Fizeau, Nice-Sophia Antipolis Univ. (France); C. Muñoz-Tuñon, Instituto de Astrofísica de Canarias (Spain); M. Sarazin, European Southern Observatory (Germany)

$70121 \mathrm{U}$ Giant Magellan Telescope site evaluation and characterization at Las Campanas Observatory [7012-65]

J. E. Thomas-Osip, G. Prieto, GMT/Las Campanas Observatory (Chile); M. Johns, M. M. Phillips, Observatories of the Carnegie Institute of Washington (United States)

$70121 \mathrm{~V}$ Inuksuit: robotic astronomical site-testing stations in the Canadian High Arctic [7012-66] E. Steinbring, B. Leckie, P. Welle, T. Hardy, Herzberg Institute of Astrophysics, National Research Council Canada (Canada); B. Cole, D. Bayne, Environment Canada (Canada); B. Croll, Univ. of Toronto (Canada); D. E. Walker, R. G. Carlberg, Cerro Tololo Inter-American Observatory (Chile); G. G. Fahlman, Herzberg Institute of Astrophysics, National Research Council Canada (Canada); B. Wallace, Defence Research and Development Canada (Canada); P. Hickson, Univ. of British Columbia (Canada)

7012 IW G-Scidar measurements of the optical turbulence with standard and high vertical resolution at Mt. Graham [7012-67]

J. Stoesz, E. Masciadri, Osservatorio Astrofisico di Arcetri (Italy); S. Hagelin, Osservatorio Astrofisico di Arcetri (Italy) and Uppsala Univ. (Sweden); F. Lascaux, Osservatorio Astrofisico di Arcetri (Italy); S. Egner, Max-Planck-Institut für Astronomie (Germany)

\section{SESSION 16 SITE TESTING AND CHARACTERIZATION II}

7012 1X Status of the Thirty Meter Telescope site selection program (Invited Paper) [7012-68] M. Schöck, TMT Observatory Corp. (United States); S. Els, Cerro Tololo Inter-American Observatory (Chile); R. Riddle, W. Skidmore, T. Travouillon, TMT Observatory Corp. (United States); R. Blum, National Optical Astronomy Observatory (United States); E. Bustos, Cerro Tololo Inter-American Observatory (Chile); P. Gillett, TMT Observatory Corp. (United States); B. Gregory, Cerro Tololo Inter-American Observatory (Chile); J. Nelson, Univ. of California, Santa Cruz (United States); A. Otárola, TMT Observatory Corp. (United States); J. Seguel, J. Vasquez, Cerro Tololo Inter-American Observatory (Chile); K. Vogiatzis, TMT Observatory Corp. (United States); D. Walker, Cerro Tololo Inter-American Observatory (Chile); L. Wang, TMT Observatory Corp. (United States)

$70121 Y$ Site selection for extremely large telescopes using the FriOWL software and global re-analysis climate data [7012-69]

E. Graham, Univ. of Bern (Switzerland); M. Sarazin, H. Kurlandczyk, European Southern Observatory (Germany); M. Neun, Univ. of Zurich (Switzerland); C. Mätzler, Univ. of Bern (Switzerland)

701212 Submillimeter observing conditions on Cerro Chajnantor [7012-70]

S. J. E. Radford, California Institute of Technology (United States); R. Giovanelli, G. E. Gull, C. P. Henderson, Cornell Univ. (United States) 
701220 Temporal variability of the seeing of TMT sites [7012-71]

T. Travouillon, TMT Observatory Corp. (United States); S. G. Els, Cerro Tololo Inter-American Observatory (Chile); R. L. Riddle, M. Schöck, A. W. Skidmore, TMT Observatory Corp. (United States); E. Bustos, J. Seguel, D. Walker, J. Vasquez, R. Blum, P. Gillett, B. Gregory, Cerro Tololo Inter-American Observatory (Chile)

701221 Combining turbulence profiles from MASS and SLODAR: a statistical study of the evolution of the seeing at Paranal [7012-72]

G. Lombardi, European Southern Observatory (Chile), Univ. of Bologna (Italy), and Bologna Astronomical Observatory, INAF (Italy); J. Navarrete, European Southern Observatory (Chile); M. Sarazin, European Southern Observatory (Germany)

\section{SESSION 17 SITE TESTING AND CHARACTERIZATION III}

701222 The Multi Aperture Scintillation Sensor (MASS) used in the site selection of the Thirty Meter Telescope (TMT) [7012-73]

S. G. Els, Cerro Tololo Inter-American Observatory (Chile) and TMT Observatory Corp. (United States); M. Schöck, TMT Observatory Corp. (United States); J. Seguel, Cerro Tololo Inter-American Observatory (Chile); W. Skidmore, TMT Observatory Corp. (United States); D. Walker, A. Tokovinin, Cerro Tololo Inter-American Observatory (Chile); V. Kornilov, Sternberg Astronomical Institute (Russia); R. Riddle, T. Travouillon, TMT Observatory Corp. (United States); E. Bustos, J. Vasquez, Cerro Tololo Inter-American Observatory (Chile); R. Blum, National Optical Astronomy Observatory (United States); B. Gregory, Cerro Tololo Inter-American Observatory (Chile); P. Gillett, TMT Observatory Corp. (United States)

701223 An analysis of light pollution at the Thirty Meter Telescope candidate sites [7012-74] R. L. Riddle, TMT Observatory Corp. (United States); D. Walker, Cerro Tololo Inter-American Observatory (Chile); M. Schöck, TMT Observatory Corp. (United States); S. G. Els, Cerro Tololo Inter-American Observatory (Chile); W. Skidmore, T. Travouillon, TMT Observatory Corp. (United States); E. Bustos, J. Seguel, J. Vasquez, Cerro Tololo Inter-American Observatory (Chile); R. D. Blum, National Optical Astronomy Observatory (United States); P. Gillett, TMT Observatory Corp. (United States); B. Gregory, Cerro Tololo Inter-American Observatory (Chile)

701224 Using All Sky Cameras to determine cloud statistics for the Thirty Meter Telescope candidate sites [7012-76] W. Skidmore, M. Schöck, TMT Observatory Corp. (United States); E. Magnier, Institute for Astronomy (United States); D. Walker, AURA/CTIO (Chile); D. Feldman, California Institute of Technology (United States); R. Riddle, TMT Observatory Corp. (United States); S. Els, AURA/CTIO (Chile); T. Travouillon, TMT Observatory Corp. (United States); E. Bustos, J. Seguel, J. Vasquez, AURA/CTIO (Chile); R. Blum, NOAO (United States); P. Gillett, TMT Observatory Corp. (United States); B. Gregory, AURA/CTIO (Chile)

701225 Optical turbulence and outer scales above Dome C in Antarctica [7012-77] H. Trinquet, A. Agabi, J. Vernin, M. Azouit, E. Aristidi, E. Fossat, Observatoire de la Côte d'Azur, CNRS, Univ. de Nice Sophia Antipolis (France) 
701226 Gattini: a multisite campaign for the measurement of sky brightness in Antarctica [7012-78] A. Moore, California Institute of Technology, Caltech Optical Observatories (United States); G. Allen, Solar Mobility (Australia); E. Aristidi, LUAN, Univ. de Nice (France); M. Ashley, Univ. of New South Wales (Australia); T. Bedding, Univ. of Sydney (Australia); C. Beichman, Jet Propulsion Lab. (United States); R. Briguglio, Univ. di Roma La Sapienza (Italy); M. Busso, Univ. di Perugia (Italy); M. Candidi, IFSI, INAF (Italy); D. Ciardi, Michelson Science Ctr. (United States); X. Cui, Nanjing Institute of Astronomical Optics \& Technology (China); G. Cutispoto, E. Distefano, Osservatorio Astrofisico di Catania, INAF (Italy); P. Espy, British Antarctic Survey (United Kingdom); J. Everett, Univ. of New South Wales (Australia); L. Feng, Purple Mountain Observatory (China); J. Hu, Z. Jiang, National Astronomical Observatories (China); S. Kenyon, Univ. of New South Wales (Australia); C. Kulesa, Steward Observatory, Univ. of Arizona (United States); J. Lawrence, Univ. of New South Wales (Australia); B. Le Roux, OAMP/LAM (France); T. Leslie, Univ. of New South Wales (Australia); Y. Li, Polar Research Institute of China (China); D. Luong-Van, A. Phillips, Univ. of New South Wales (Australia); W. Qin, Polar Research Institute of China (China); R. Ragazzoni, Osservatorio Astrofisico di Padova, INAF (Italy); R. Riddle, TMT project, California Institute of Technology (United States); L. Sabbatini, Univ. di Roma La Sapienza (Italy); P. Salinari, Osservatorio Astrofisico di Arcetri, INAF (Italy); W. Saunders, Anglo-Australian Observatory (Australia); Z. Shang, Tianjin Normal Univ. (China); D. Stello, Univ. of Sydney (Australia); J. Storey, Univ. of New South Wales (Australia); B. Sun, Polar Research Institute of China (China); N. Suntzeff, Cerro Tololo Inter-American Observatory, National Optical Astronomy Observatory (Chile) and Texas A\&M Univ. (United States); M. Taylor, Univ. of New South Wales (Australia); G. Tosti, Univ. di Perugia (Italy); N. Tothill, Univ. of Exeter (United Kingdom); T. Travouillon, California Institute of Technology, Caltech Optical Observatories (United States); G. Van Belle, European Southern Observatory (Germany); K. Von Braun, Michelson Science Ctr. (United States); L. Wang, J. Yan, Purple Mountain Observatory (China); H. Yang, Polar Research Institute of China (China); X. Yuan, Nanjing Institute of Astronomical Optics \& Technology, National Astronomical Observatories (China); Z. Zhu, Purple Mountain Observatory (China); X. Zhou, National Astronomical Observatories (China)

701227 The PLATO Antarctic site testing observatory [7012-79]

J. S. Lawrence, Univ. of New South Wales (Australia); G. R. Allen, Solar Mobility (Australia); M. C. B. Ashley, C. Bonner, Univ. of New South Wales (Australia); S. Bradley, Univ. of Auckland (New Zealand); X. Cui, Nanjing Institute of Astronomical Optics Technology (China); J. R. Everett, Univ. of New South Wales (Australia); L. Feng, Purple Mountain Observatory (China); X. Gong, Nanjing Institute of Astronomical Optics Technology (China); S. Hengst, Univ. of New South Wales (Australia); J. Hu, Z. Jiang, National Astronomical Observatory of China (China); C. A. Kulesa, Univ. of Arizona (United States); Y. Li, Polar Research Institute of China (China); D. Luong-Van, Univ. of New South Wales (Australia); A. M. Moore, California Institute of Technology (United States); C. Pennypacker, Univ. of California, Berkeley (United States); W. Qin, Polar Research Institute of China (China); R. Riddle, Thirty Meter Telescope Observatory Corp. (United States); Z. Shang, Tianjin Normal Univ. (China); J. W. V. Storey, Univ. of New South Wales (Australia); B. Sun, Polar Research Institute of China (China); N. Suntzeff, Texas A\&M Univ. (United States); N. F. H. Tothill, Univ. of Exeter (United Kingdom); T. Travouillon, California Institute of Technology (United States); C. K. Walker, Univ. of Arizona (United States); L. Wang, Purple Mountain Observatory (China) and Texas A\&M Univ. (United States); J. Yan, Purple Mountain Observatory (China) and National Astronomical Observatory of China (China); J. Yang, Purple Mountain Observatory (China); H. Yang, Polar Research Institute of China (China); D. York, Univ. of Chicago (United States); X. Yuan, Nanjing Institute of Astronomical Optics Technology (China); X. G. Zhang, Purple Mountain Observatory (China); Z. Zhang, 
Polar Research Institute of China (China); X. Zhou, National Astronomical Observatory of China (China); Z. Zhu, Purple Mountain Observatory (China)

701228 A 30-m submillimeter telescope with active reflector [7012-80]

$X$. Cui, National Astronomical Observatories, Nanjing Institute of Astronomical Optics and Technology (China); D. Su, National Astronomical Observatories, Nanjing Institute of Astronomical Optics and Technology (China) and Univ. of Nanjing (China); Y. Wang, D. Yang, G. Li, National Astronomical Observatories, Nanjing Institute of Astronomical Optics and Technology (China)

701229 A 40-cm infra-red telescope in Antarctica [7012-81]

C. Murata, T. Ichikawa, R. G. Lundock, Y. Taniguchi, H. Okita, Astronomical Institute, Tohoku Univ. (Japan)

7012 2A ACWI: an experiment to image the Cosmic Web from Antarctica [7012-82]

A. M. Moore, C. Martin, Caltech Institute of Technology (United States); N. C. Maitless, Harvard Graduate School of Design (United States); T. Travouillon, Caltech Institute of Technology (United States)

7012 2B Toward a large telescope facility for submm/FIR astronomy at Dome C [7012-83] G. A. Durand, V. Minier, P.-O. Lagage, E. Daddi, S. El Khouloudi, N. Schneider-Bontemps, M. Talvard, CEA, DSM, IRFU, Service d'Astrophysique (France); C. Veyssière, G. A. Durand, C. Walter, CEA, DSM, IRFU, SIS (France); L. Sabbatini, Univ. of Rome La Sapienza (Italy); Z. Challita, Lab. H. Fizeau, CNRS, OCA, Univ. de Nice (France) and IPEV (France); J. W. V. Storey, Univ. of New South Wales (Australia); P. Calisse, Cardiff Univ. (United Kingdom); A. Pierre, IPEV (France); M. Busso, Univ. di Perugia (Italy)

7012 2C The Antarctica Wide-field High-resolution Infrared Telescope (WHITE) [7012-84] D. Burgarella, B. Le Roux, M. Langlois, G. Lemaître, Observatoire Astronomique Marseille Provence, Lab. d'Astrophysique de Marseille, Univ. d'Aix-Marseille, CNRS (France); T. Fusco, ONERA-DOTA (France); M. Ferrari, Observatoire Astronomique Marseille Provence, Lab. d'Astrophysique de Marseille, Univ. d'Aix-Marseille, CNRS (France)

7012 2D Antarctic Schmidt Telescopes (AST3) for Dome A [7012-85]

X. Cui, X. Yuan, X. Gong, National Astronomical Observatories, Nanjing Institute of Astronomical Optics \& Technology (China) and Chinese Ctr. for Antarctic Astronomy (China)

\section{SESSION 20 TELESCOPE STRUCTURES}

7012 2E Seismic hazard: analysis and design of large ground-based telescopes [7012-86] F. W. Kan, J. Antebi, Simpson Gumpertz \& Heger (United States)

7012 2F The Giant Magellan Telescope (GMT): structure design update [7012-87] S. Gunnels, Paragon Engineering (United States); F. Kan, A. Sarawit, Simpson Gumpertz \& Heger (United States) 
$70122 \mathrm{G}$ TMT telescope structure system: design and development progress report [7012-88] K. Szeto, S. Roberts, Herzberg Institute of Astrophysics, National Research Council Canada (Canada); M. Gedig, G. Austin, C. Lagally, S. Patrick, D. Tsang, Empire Dynamic Structures (Canada); D. MacMynowski, M. Sirota, L. Stepp, Thirty Meter Telescope Project (United States); P. M. Thompson, Systems Technology, Inc. (United States)

$70122 \mathrm{H} \quad$ Photogrammetry measurement of the AMiBA 6-meter platform [7012-89]

Y. D. Huang, Academia Sinica, Institute of Astronomy and Astrophysics (Taiwan); P. Raffin, M.-T. Chen, P. Altamirano, P. Oshiro, ASIAA (Taiwan)

701221 Design concepts for primary mirror support structures of large telescopes for optical and submillimeter astronomy [7012-90]

C. L. Stutzki, H. Tamai, Stutzki Engineering, Inc. (United States); T. A. Sebring, Cornell Univ. (United States)

\section{POSTER SESSION: INTEGRATION, COMMISSIONING, AND EARLY RESULTS}

7012 2J Vibration measurements at the Large Binocular Telescope (LBT) [7012-92]

M. Brix, V. Naranjo, Max-Planck-Institut für Astronomie (Germany); U. Beckmann, MaxPlanck-Institut für Radioastronomie (Germany); R. Bertram, LBT Observatory, Univ. of Arizona (United States); T. Bertram, Univ. Köln (Germany); J. Brynnel, LBT Observatory, Univ. of Arizona (United States); S. Egner, W. Gaessler, T. M. Herbst, M. Kuerster, R. R. Rohloff, MaxPlanck-Institut für Astronomie (Germany); S. Rost, Univ. Köln (Germany); J. Schmidt, MaxPlanck-Institut für Astronomie (Germany)

\section{POSTER SESSION: NEW FACILITIES AND APPROACHES}

$70122 \mathrm{~K} \quad$ VST primary mirror active optics power distribution system [7012-94]

C. Molfese, VSTceN, INAF (Italy); A. Busatta, EIE-European Industrial Engineering (Italy)

7012 2L The Earthshine Telescope Project [7012-95]

M. Owner-Petersen, T. Andersen, A. Ardeberg, Lund Observatory (Sweden); P. Thejll,

H. Gleisner, Danish Meteorological Institute (Denmark)

701220 Observatorio UC at Santa Martina: a small observing facility operated by PUC [7012-99] M. Baffico, Pontificia Univ. Catolica de Chile (Chile); G. Avila, D. Baade, E. Bendek, C. Guirao, European Southern Observatory (Chile); O. Gonzalez, P. Marchant, V. Salas, I. Toledo, S. Vasquez, L. Vanzi, Pontificia Univ. Catolica de Chile (Chile)

7012 2P Transient optical sky survey automated telescope system [7012-100]

E. Hadjiyska, P. Lubin, Univ. of California, Santa Barbara (United States); S. Taylor, Raytheon Co. (United States); G. Hughes, California Polytechnic State Univ. (United States)

$70122 Q \quad$ Gemini primary mirror in-situ wash [7012-101]

T. Vucina, M. Boccas, C. Araya, Gemini Observatory (Chile); C. Ah Hee, C. Cavedoni, Gemini Observatory (United States)

7012 2R The Pan-STARRS PS4 telescope suite [7012-102] J. S. Morgan, W. Burgett, Pan-STARRS, Univ. of Hawaii (United States); J. U. Teran, M3 Engineering \& Technology Corp. (United States) 
701225 Ground-based complex for detection and investigation of fast optical transients in wide field [7012-104]

E. Molinari, Osservatorio Astronomico di Brera (Italy); G. Beskin, Special Astrophysical Observatory (Russia); S. Bondar, Institute for Precise Instrumentation (Russia); S. Karpov, V. Plokhotnichenko, V. de-Bur, Special Astrophysical Observatory (Russia); G. Greco, C. Bartolini, A. Guarnieri, A. Piccioni, Univ. degli Studi di Bologna (Italy)

7012 2T The University of Tokyo Atacama 1.0-m telescope [7012-105]

S. Sako, Institute of Astronomy, Univ. of Tokyo (Japan); T. Aoki, Kiso Observatory, Univ. of Tokyo (Japan); M. Doi, T. Handa, K. Kawara, K. Kohno, T. Minezaki, N. Mitani, T. Miyata, K. Motohara, Institute of Astronomy, Univ. of Tokyo (Japan); T. Soyano, Kiso Observatory, Univ. of Tokyo (Japan); T. Tanabe, M. Tanaka, Institute of Astronomy, Univ. of Tokyo (Japan); K. Tarusawa, Kiso Observatory, Univ. of Tokyo (Japan); Y. Yoshii, Institute of Astronomy, Univ. of Tokyo (Japan); L. Bronfman, M. T. Ruiz, Univ. of Chile, Santiago (Chile)

$70122 \mathrm{U}$ High-resolution image reconstruction technique applied to the optical testing of ground-based astronomical telescopes [7012-106]

Z. Jin, J. Lin, Z. Liu, Yunnan Astronomical Observatory (China)

7012 2W CCD charge transfer efficiency test with the new DES clock board [7012-108]

J. Campa, Ctr. de Investigaciones Energeticas, Medioambientales y Tecnologicas (Spain); L. Cardiel, Institut de Física d'Altes Energies (Spain); J. Castilla, J. De Vicente, Ctr. de Investigaciones Energeticas, Medioambientales y Tecnologicas (Spain); J. Estrada, Fermi National Accelerator Lab. (United States); I. Karliner, Univ. of Illinois at Urbana-Champaign (United States); D. Kubik, Fermi National Accelerator Lab. (United States); G. Martinez, Ctr. de Investigaciones Energeticas, Medioambientales y Tecnologicas (Spain); T. Shaw, W. Stuermer, Fermi National Accelerator Lab. (United States)

\section{Part Three}

7012 2X New robotic telescopes by Halfmann-Teleskoptechnik GmbH and Tuparev Technologies Inc. [7012-109] K. Bischoff, Halfmann Teleskoptechnik (Germany); F. V. Hessman, Institut für Astrophysik (Germany); G. Tuparev, E. Atanasova, P. Pessev, Tuparev Technologies (Netherlands)

$70122 Y$ The Gemini MCAO infrastructure: laser service enclosure and support structure [7012-110] C. P. Cavedoni, Gemini Observatory (United States); S. Bombino, M. Sheehan, S. Karewicz, S. Hardash, Gemini Observatory Northern Operations Ctr. (United States); G. Perez, P. Collins, C. d'Orgeville, M. Boccas, D. Maltes, G. Gausachs, R. Rogers, Gemini Observatory Southern Operations Ctr. (Chile)

$70122 Z$ The impact of seismicity on high angular resolution astronomy: the case of El Teide Observatory, Canary Islands [7012-111]

A. Eff-Darwich, Univ. de La Laguna (Spain) and Instituto de Astrofísica de Canarias (Spain); B. García-Lorenzo, Instituto de Astrofísica de Canarias (Spain); L. Bonatto, Institute of Earth Sciences, Jaume Almera (Spain); L. E. Hernández-Gutierrez, Consejería de Obras Públicas y Transportes del Gobierno de Canarias (Spain); R. Viñas, J. A. Rodriguez-Losada, Univ. de La Laguna (Spain); M. J. Blanco, Instituto Geográfico Nacional (Spain); C. Muñoz-Tuñón, Instituto de Astrofísica de Canarias (Spain) 
701230 Evaluation of thermal control coatings exposed to ambient weather conditions at Haleakalā High Altitude Observatory [7012-112]

L. Phelps, National Solar Observatory (United States)

701231 The feasibility of large refracting telescopes for solar coronal research [7012-113] P. G. Nelson, S. Tomczyk, D. F. Elmore, D. J. Kolinski, High Altitude Observatory (United States)

701232 The participation of the Instituto de Astrofísica de Canarias in the European Solar Telescope [7012-114]

M. Collados, A. Calcines, J. J. Díaz, F. Gracia, C. Grivel-Gelly, R. López, H. Mangharam, E. Páez, A. Pérez, J. L. Rasilla, L. F. Rodríguez, J. Sánchez-Capuchino, H. Socas-Navarro, Instituto de Astrofísica de Canarias (Spain)

701233 Advanced Technology Solar Telescope M1 thermal control system design, modeling, and prototype testing [7012-115]

E. Hansen, S. Bulau, L. Phelps, AURA, National Solar Observatory (United States)

701234 Assessment of local seeing within a telescope lab environment [7012-116]

R. Biérent, Ecole Nationale Supérieure de Physique de Grenoble (France); T. Rimmele,

J. Marino, National Solar Observatory (United States)

701235 Optomechanical and thermal design of the Multi-Application Solar Telescope for USO [7012-117]

S. Denis, P. Coucke, E. Gabriel, C. Delrez, AMOS SA (Belgium); P. Venkatakrishnan, Udaipur Solar Observatory (India)

POSTER SESSION: ALMA

701236 The European ALMA production antennas: new drive applications for better performances and low cost management [7012-119]

L. Giacomel, C. Manfrin, G. Marchiori, European Industrial Engineering (Italy)

\section{POSTER SESSION: RADIO TELESCOPES AND THEIR TECHNOLOGY}

701237 Replacement of the Green Bank Telescope azimuth track [7012-120]

R. Anderson, A. Symmes, D. Egan, National Radio Astronomy Observatory (United States)

701238 Improving the service life of the 100-meter Green Bank Telescope azimuth track [7012-121] A. Symmes, R. Anderson, D. Egan, National Radio Astronomy Observatory (United States)

701239 Proposed design concepts of the FAST focus cabin suspension [7012-123]

H. J. Kärcher, MT Mechatronics GmbH (Germany); H. Li, J. Sun, R. Nan, National Astronomical Observatories (China); M. Lazanowski, FNM, TU Darmstadt (Germany); S. Kern, B. Strah, F. Fomi, MiM, TU Darmstadt (Germany) 
7012 3A Design and performances of the Shack-Hartmann sensor within the Active Phasing Experiment [7012-124]

R. Mazzoleni, F. Gonté, I. Surdej, C. Araujo, R. Brast, F. Derie, P. Duhoux, C. Dupuy, C. Frank, R. Karban, L. Noethe, N. Yaitskova, European Organisation for Astronomical Research in the Southern Hemisphere (Germany)

7012 3B ASM: a scaled down Active Segmented Mirror for the Active Phasing Experiment [7012-127] C. Dupuy, F. Gonté, C. Frank, European Southern Observatory (Germany)

$70123 \mathrm{C}$ Large capture range cophasing with the Liquid Crystal Tunable Filter [7012-128] M. Bonaglia, E. Pinna, F. Quiros-Pacheco, A. Puglisi, S. Esposito, Osservatorio Astrofisico di Arcetri (Italy)

7012 3D The Pyramid Phasing Sensor (PYPS) [7012-129]

E. Pinna, F. Quirós-Pacheco, S. Esposito, A. Puglisi, P. Stefanini, Osservatorio Astrofisico di Arcetri (Italy)

7012 3E Double segmentation control with a single phasing sensor [7012-130]

F. Quirós-Pacheco, E. Pinna, S. Esposito, A. Riccardi, Osservatorio Astrofisico di Arcetri (Italy)

$70123 \mathrm{~F}$ Development of a novel actuator concept for position control of segmented mirrors of ELT [7012-131]

H. Janssen, R. Geurink, M. Teuwen, B. v. Bree, Janssen Precision Engineering B.V. (Netherlands)

$70123 G$ SALT segmented primary mirror: commissioning capacitive edge sensing system and performance comparison with inductive sensor [7012-132]

S. Buous, J. Menzies, H. Gajjar, Southern African Large Telescope, South African

Astronomical Observatory (South Africa)

$70123 \mathrm{H} \quad$ Progress of LAMOST wavefront sensing [7012-133]

Y. Zhang, National Astronomical Observatories, Nanjing Institute of Astronomical Optics and Technology (China)

\section{POSTER SESSION: FUTURE GIANT TELESCOPES}

$701231 \quad$ Ultra low wind resistance enclosure for a $100 \mathrm{~m}$ telescope [7012-134]

T. D. Ditto, DeWitt Brothers Tool Co., Inc. (United States); J. M. Ritter, Institute for Astronomy, Univ. of Hawaii (United States)

7012 3J A preliminary design for the E-ELT enclosure [7012-179]

J. Cross, J. Lyle, Arup (United Kingdom); M. Schneermann, European Southern Observatory (Germany) 
POSTER SESSION: TELESCOPE ALIGNMENT AND CONTROL

7012 3K Cerro Tololo Inter-American Observatory, Victor M. Blanco 4-m Telescope: an upgrade to the telescope control system [7012-135]

T. M. C. Abbott, G. Schumacher, M. Warner, E. Mondaca, R. E. Schmidt, R. Cantarutti, M. Martinez, O. Estay, F. Delgado, A. Walker, National Optical Astronomy Observatories (United States)

$70123 \mathrm{~L} \quad$ VST telescope azimuth and altitude axes control hardware [7012-136]

C. Molfese, VSTceN, INAF (Italy); P. Schipani, Osservatorio Astronomico di Capodimonte, INAF (Italy); M. Capaccioli, VSTceN, INAF (Italy) and Univ. degli Studi Federico II (Italy); G. Sedmak, Univ. degli Studi di Trieste (Italy); D. Mancini, Osservatorio Astronomico di Capodimonte, INAF (Italy); S. D'Orsi, VSTceN, INAF (Italy)

$70123 \mathrm{M} \quad$ VST hydrostatic bearing system control hardware [7012-137]

C. Molfese, VSTceN, INAF (Italy); P. Schipani, D. Mancini, Osservatorio Astronomico di Capodimonte, INAF (Italy); S. D'Orsi, VSTceN, INAF (Italy)

$70123 \mathrm{~N} \quad$ Adaptive periodic error correction for Heidenhain tape encoders [7012-138] M. Warner, Cerro Tololo Inter-American Observatory (Chile); V. Krabbendam, LSST Telescope Project (United States); G. Schumacher, Cerro Tololo Inter-American Observatory (Chile)

701230 One direct drive system for telescope [7012-140]

C. Ren, National Astronomical Observatories, Nanjing Institute of Astronomical Optics \& Technology (China) and Graduate School of the Chinese Academy of Sciences (China); Z. Zhang, D. Wang, National Astronomical Observatories, Nanjing Institute of Astronomical Optics \& Technology (China); X. Shuai, W. Hu, National Astronomical Observatories, Nanjing Institute of Astronomical Optics \& Technology (China) and Graduate School of the Chinese Academy of Sciences (China); G. Wang, National Astronomical Observatories, Nanjing Institute of Astronomical Optics \& Technology (China); Z. Zhu, Nanjing Univ. of Aeronautics and Astronautics (China)

$70123 \mathrm{P}$ The use of primary mirrors as Hartmann masks for in situ alignment of segmented mirror telescopes [7012-141]

S. M. Crawford, South African Astronomical Observatory (South Africa); M. Wells, UK Astronomy Technology Ctr., Royal Observatory (United Kingdom); H. Gajjar, South African Astronomical Observatory (South Africa)

\section{POSTER SESSION: AIRBORNE TELESCOPES}

$70123 Q \quad$ Sub-arcsecond pointing for balloon-borne telescopes [7012-142]

A. Kraut, K. Swartzlander, E. Wong, G. Orr, T. Wimer, Harvey Mudd College (United States); Y. Nakaya, Kogakuin Univ. (Japan); M. Bullock, E. Young, Southwest Research Institute (United States); P. Little, Harvey Mudd College (United States)

$70123 R \quad$ THz balloon-borne telescope: Tera-GATE project [7012-143]

T. Kamizuka, The Univ. of Tokyo (Japan) and Institute of Space and Astronautical Science, Japan Aerospace Exploration Agency (Japan); H. Kataza, K. Watanabe, T. Wada, H. Murakami, Institute of Space and Astronautical Science, Japan Aerospace Exploration Agency (Japan) 
701235 Optical design of image stabilizing Cassegrain-like balloon-borne telescope [7012-144] H. Kataza, Institute of Space and Astronomical Science, Japan Aerospace Exploration Agency (Japan); T. Kamizuka, Institute of Space and Astronomical Science, Japan Aerospace Exploration Agency (Japan) and The Univ. of Tokyo (Japan); K. Watanabe, H. Murakami, T. Wada, Institute of Space and Astronomical Science, Japan Aerospace Exploration Agency (Japan)

$70123 \mathrm{~T}$ The SOFIA Telescope: preparing for early science [7012-145]

H. J. Kärcher, MT Mechatronics GmbH (Germany); A. Krabbe, I. Physikalisches Institut, Univ. zu Köln (Germany); T. Wegmann, Deutsches SOFIA Institut, Univ. Stuttgart (Germany)

\section{POSTER SESSION: SITE TESTING AND CHARACTERIZATION}

$70123 \mathrm{U}$ Calibration of the Boltwood Cloud Sensor [7012-146]

J. Marchant, R. J. Smith, I. A. Steele, Astrophysics Research Institute, Liverpool John Moores Univ. (United Kingdom)

$70123 \mathrm{~V}$ Estimating local seeing at the DCT facility [7012-147]

D. Blanco, NOAO (United States) and College of Optical Sciences, The Univ. of Arizona (United States)

7012 3W LSST IR camera for cloud monitoring and observation planning [7012-148]

J. Sebag, V. L. Krabbendam, C. F. Claver, J. Andrew, J. D. Barr, National Optical Astronomy Observatory (United States); D. Klebe, Denver Museum of Nature and Science (United States)

$70123 X \quad$ First results from turbulence profiling with SODAR at Subaru Telescope [7012-149] F. Uraguchi, N. Takato, Subaru Telescope, National Astronomical Observatory of Japan (United States); I. Iwata, H. Koyano, Okayama Astrophysical Observatory, National Astronomical Observatory of Japan (Japan); Y. Mikami, National Astronomical Observatory of Japan (Japan)

$70123 Z$ Optimizing atmospheric correction at the JCMT using a $183 \mathrm{GHz}$ water vapour radiometer [7012-151]

J. T. Dempsey, P. Friberg, Joint Astronomy Ctr. (United States)

701240 The bistatic LGS scheme for sodium layer height characterization (based on the Teide Observatory experimental setup) [7012-152]

J. A. Castro-Almazán, J. J. Fuensalida, Á. Alonso, S. Chueca, Instituto de Astrofísica de Canarias (Spain)

701241 A study of the aerosol extinction from ground-based dust measurements at ORM [7012-154] G. Lombardi, Univ. of Bologna (Italy), INAF, Bologna Astronomical Observatory (Italy), and European Southern Observatory (Chile); V. Zitelli, INAF, Bologna Astronomical Observatory (Italy); S. Ortolani, Univ. of Padova (Italy); M. Pedani, Large Binocular Telescope Observatory, Univ. of Arizona (United States); A. Ghedina, Fundación Galileo Galilei and Telescopio Nazionale Galileo (Spain) 
701243 Site evaluations of the summit of Co. Chajnantor for infrared observations [7012-156] T. Miyata, K. Motohara, S. Sako, M. Tanaka, T. Minezaki, N. Mitani, Institute of Astronomy, The Univ. of Tokyo (Japan); T. Aoki, T. Soyano, Kiso Observatory, Institute of Astronomy, The Univ. of Tokyo (Japan); T. Tanabe, K. Kawara, K. Kohno, Institute of Astronomy, The Univ. of Tokyo (Japan); K. Tarusawa, Kiso Observatory, Institute of Astronomy, The Univ. of Tokyo (Japan); T. Handa, M. Doi, Y. Yoshii, Institute of Astronomy, The Univ. of Tokyo (Japan); H. Ezawa, National Astronomical Observatory of Japan (Japan); L. Bronfman, M. T. Ruiz, Univ. of Chile (Chile)

701244 Seeing environment at a $5640 \mathrm{~m}$ altitude of Co. Chajnantor in northern Chile [7012-157] K. Motohara, Institute of Astronomy, Univ. of Tokyo (Japan); T. Aoki, Kiso Observatory, Univ. of Tokyo (Japan); S. Sako, Institute of Astronomy, Univ. of Tokyo (Japan); T. Soyano, Kiso Observatory, Univ. of Tokyo (Japan); M. Doi, M. Tanaka, T. Tanabe, T. Handa, N. Mitani, T. Minezaki, T. Miyata, K. Kawara, K. Kohno, Institute of Astronomy, Univ. of Tokyo (Japan); K. Tarusawa, Kiso Observatory, Univ. of Tokyo (Japan); Y. Yoshii, Institute of Astronomy, Univ. of Tokyo (Japan); L. Bronfman, M. T. Ruiz, Univ. of Chile (Chile); F. Uraguchi, N. Takato, Subaru Telescope, National Astronomical Observatory of Japan (United States)

701245 Design and performance of the Paranal cute-SCIDAR instrument for real-time turbulence profiles measurements [7012-161]

H. Vázquez Ramió, J. M. Delgado, M. Reyes, J. J. Fuensalida, E. Hernández, M. Núñez, B. M. García-Lorenzo, M. A. C. Rodríguez-Hernández, Instituto de Astrofísica de Canarias (Spain); J. Vernin, Univ. de Nice Sophia Antipolis (France)

701247 TOE-The Onduline Experiment: a new kind of wavefront sensor to characterize astronomical sites for Extremely Large Telescopes [7012-163]

C. Metti, G. Gentile, M. Dima, J. Farinato, C. Arcidiacono, A. Baruffolo, V. Viotto, Astronomical Observatory of Padova, INAF (Italy); E. Diolaiti, Astronomical Observatory of Bologna, INAF (Italy); R. Ragazzoni, Astronomical Observatory of Padova, INAF (Italy)

\section{POSTER SESSION: SITE TESTING IN ANTARCTICA}

701248 First photometric properties of Dome C, Antarctica [7012-164]

M. Chadid, J. Vernin, F. Jeanneaux, D. Mekarnia, H. Trinquet, UMR 6225, Observatoire de la Cote d'Azur, Univ. de Nice Sophia, Antipolis (France)

701249 Pre-HEAT: submillimeter site testing and astronomical spectra from Dome A, Antarctica [7012-165]

C. A. Kulesa, C. K. Walker, M. Schein, D. Golish, Univ. of Arizona (United States); N. Tothill, Univ. of Exeter (United Kingdom); P. Siegel, S. Weinreb, G. Jones, J. Bardin, California Institute of Technology (United States); K. Jacobs, Univ. of Cologne (Germany); C. L. Martin, Oberlin College (United States); J. Storey, M. Ashley, J. Lawrence, D. Luong-Van, J. Everett, Univ. of New South Wales (Australia); L. Wang, Texas A\&M Univ. (United States); L. Feng, Z. Zhu, J. Yan, J. Yang, X.-G. Zhang, Purple Mountain Observatory (China); X. Cui, X. Yuan, Nanjing Institute of Astronomical Optics Technology (China); J. Hu, Z. Xu, Z. Jiang, National Astronomical Observatory of China (China); H. Yang, Y. Li, B. Sun, W. Qin, Polar Research Institute of China (China); Z. Shang, Tianjin Normal Univ. (China) 
7012 4A Comparison of the atmospheric properties above Dome A, Dome C, and the South Pole [7012-166]

S. Hagelin, Osservatorio Astrofisico di Arcetri, INAF (Italy) and Uppsala Univ. (Sweden);

E. Masciadri, F. Lascaux, J. Stoesz, Osservatorio Astrofisico di Arcetri, INAF (Italy)

7012 4B Sampling the ground layer of the atmosphere at Dome C using fast sonic-anemometers [7012-167]

T. Travouillon, California Institute of Technology (United States); E. Aristidi, E. Fossat, UMR 6526, Univ. of Nice-Sophia Antipolis (France); J. S. Lawrence, Univ. of New South Wales (Australia); D. Mekarnia, UMR 6526, Univ. of Nice-Sophia Antipolis (France); A. M. Moore, California Institute of Technology (United States); A. W. Skidmore, TMT Observatory Corp. (United States); J. W. V. Storey, Univ. of New South Wales (Australia)

7012 4C Wide-field performance gradient at a mid-latitude site and at Dome C [7012-168] J. Stoesz, E. Masciadri, Osservatorio Astrofisico di Arcetri (Italy); S. Hagelin, Osservatorio Astrofisico di Arcetri (Italy) and Uppsala Univ. (Sweden); F. Lascaux, Osservatorio Astrofisico di Arcetri (Italy)

7012 4D Meso-Nh simulations of the atmospheric flow above the Internal Antarctic Plateau [7012-169]

F. Lascaux, E. Masciadri, Osservatorio Astrofisico di Arcetri, INAF (Italy); S. Hagelin, Osservatorio Astrofisico di Arcetri, INAF (Italy) and Uppsala Univ. (Sweden); J. A. Stoesz, Osservatorio Astrofisico di Arcetri, INAF (Italy)

7012 4E PLATO power: a robust low environmental impact power generation system for the Antarctic plateau [7012-170]

S. Hengst, Univ. of New South Wales (Australia); G. R. Allen, Solar Mobility Pty Ltd. (Australia); M. C. B. Ashley, J. R. Everett, J. S. Lawrence, D. M. Luong-Van, J. W. V. Storey, Univ. of New South Wales (Australia)

\section{POSTER SESSION: NEW ANTARCTIC FACILITIES}

7012 4F PILOT: a wide-field telescope for the Antarctic plateau [7012-171]

W. Saunders, P. Gillingham, A. McGrath, R. Haynes, J. Brzeski, Anglo-Australian Observatory (Australia); J. Storey, J. Lawrence, Univ. of New South Wales (Australia)

7012 4G Chinese Small Telescope Array (CSTAR) for Antarctic Dome A [7012-172]

X. Yuan, X. Cui, Nanjing Institute of Astronomical Optics \& Technology (China) and Chinese Ctr. for Antarctic Astronomy (China); G. Liu, F. Zhai, National Astronomical Observatories, Nanjing Institute of Astronomical Optics \& Technology (China); X. Gong, National Astronomical Observatories, Nanjing Institute of Astronomical Optics \& Technology (China) and Chinese Ctr. for Antarctic Astronomy (China); R. Zhang, L. Xia, National Astronomical Observatories, Nanjing Institute of Astronomical Optics \& Technology (China); J. Hu, National Astronomical Observatory (China) and Chinese Ctr. for Antarctic Astronomy (China); J. S. Lawrence, Univ. of New South Wales (Australia); J. Yan, National Astronomical Observatory (China) and Chinese Ctr. For Antarctic Astronomy (China); J. W. V. Storey, Univ. of New South Wales (Australia); L. Wang, L. Feng, Purple Mountain Observatory (China) and Chinese Ctr. for Antarctic Astronomy (China); M. C. B. Ashley, Univ. of New South Wales (Australia); X. Zhou, Z. Jiang, National Astronomical Observatory (China) and Chinese Ctr. for Antarctic Astronomy (China); Z. Zhu, Purple Mountain Observatory (China) and Chinese Ctr. for Antarctic Astronomy (China) 
70124 Design of the Discovery Channel Telescope mount [7012-174]

D. T. Finley, C. Squires, B. A. McCreight, General Dynamics SATCOM Technologies (United States); B. W. Smith, T. Chylek, A. Venetiou, Lowell Observatory (United States)

7012 4J TMT telescope structure system: seismic analysis and design [7012-175]

D. Tsang, G. Austin, M. Gedig, C. Lagally, Empire Dynamic Structures (Canada); K. Szeto, Hertzberg Institute of Astrophysics, National Research Council Canada (Canada);

G. Sagals, Association of Canadian Univs. for Research in Astronomy (Canada); L. Stepp, Thirty Meter Telescope Project (United States)

7012 4K Large telescopes and the art of bridge building [7012-176]

H. J. Kärcher, MT Mechatronics GmbH (Germany)

$70124 \mathrm{~L} \quad$ Structural ropes development for the E-ELT structure [7012-177]

E. Pajuelo, J. R. Gómez, B. Ronquillo, MEDIA Consultores (Spain); E. Brunetto, F. Koch, European Southern Observatory (Germany)

7012 4M Design, manufacture, measurement, and installation of the gross mirror cell of the LAMOST Schmidt plate [7012-178]

D. Yang, K. Chen, Y. Liang, National Astronomical Observatories, Nanjing Institute of Astronomical Optics \& Technology (China); S. Wang, National Astronomical Observatories (China); G. Zhou, National Astronomical Observatories, Nanjing Institute of Astronomical Optics \& Technology (China)

Author Index 
Downloaded From: https://www.spiedigitallibrary.org/conference-proceedings-of-spie on 26 Apr 2023

Terms of Use: https://www.spiedigitallibrary.org/terms-of-use 


\title{
Conference Committee
}

\author{
Symposium Chairs
}

Mark C. Clampin, NASA Goddard Space Flight Center(United States)

Alan F. M. Moorwood, European Southern Observatory (Germany)

Symposium Cochairs

Masanori lye, National Astronomical Observatory of Japan (Japan)

Douglas A. Simons, Gemini Observatory (United States)

Conference Chairs

Larry M. Stepp, Thirty Meter Telescope Project (United States)

Roberto Gilmozzi, European Southern Observatory (Germany)

Program Committee

Torben E. Andersen, Lund Observatory, Lund University (Sweden)

Anthony J. Beasley, Atacama Large Millimeter Array (Chile)

F. Javier Castro López-Tarruella, Grantecan SA (Spain)

Jean-Gabriel Cuby, Observatoire Astronomique de MarseilleProvence (France)

Xiangqun Cui, Nanjing Institute of Astronomical Optics \& Technology (China)

Philippe Dierickx, European Southern Observatory (Germany)

Richard F. Green, Large Binocular Telescope Observatory, The University of Arizona (United States)

Masanori lye, National Astronomical Observatory of Japan (Japan)

Frank W. Kan, Simpson Gumpertz \& Heger, Inc. (United States)

Alistair M. McPherson, United Kingdom Astronomy Technology Centre (United Kingdom)

Simon J. Radford, California Institute of Technology (United States)

Thomas A. Sebring, Cornell Caltech Atacama Telescope Project (United States)

Jason Spyromilio, European Southern Observatory (Germany)

Donald W. Sweeney, LSST Corporation (United States)

Jeremy J. Wagner, Association of Universities for Research in Astronomy (United States) 
Session Chairs

1 Project Reviews

Roberto Gilmozzi, European Southern Observatory (Germany)

2 Integration, Commissioning, and Early Results

Richard F. Green, The University of Arizona (United States)

3 New Facilities and Approaches I

Xiangqun Cui, Nanjing Institute of Astronomical Optics \& Technology (China)

$4 \quad$ Solar Telescopes

Simon J. E. Radford, California Institute of Technology (United States)

5 ALMA

Thomas A. Sebring, Cornell University (United States)

6 Radio Telescopes and Their Technology

F. Javier Castro López-Tarruella, Instituto de Astrofísica de Canarias (Spain)

$7 \quad$ Wind Loading on Telescopes

F. Javier Castro López-Tarruella, Instituto de Astrofísica de Canarias (Spain)

8 Segmented Mirror Alignment and Phasing I

Philippe Dierickx, European Southern Observatory (Germany)

9 Segmented Mirror Alignment and Phasing II

Jean-Gabriel Cuby, Observatoire Astronomique de Marseille-

Provence (France)

10 Future Giant Telescopes I

Donald W. Sweeney, LSST Corporation (United States)

11 Future Giant Telescopes II

Masanori lye, National Astronomical Observatory of Japan (Japan)

12 Future Giant Telescopes III

Torben E. Andersen, Lund Observatory, Lund University (Sweden)

13 Telescope Alignment and Control I

Jason Spyromilio, European Southern Observatory (Germany) 


\section{Airborne Telescopes}

Roberto Gilmozzi, European Southern Observatory (Germany)

15 Site Testing and Characterization I

Richard F. Green, The University of Arizona (United States)

16 Site Testing and Characterization II

Larry M. Stepp, Thirty Meter Telescope Project (United States)

17 Site Testing and Characterization III

Frank W. Kan, Simpson Gumpertz \& Heger, Inc. (United States)

18 Site Testing in Antarctica

Frank W. Kan, Simpson Gumpertz \& Heger, Inc. (United States)

19 New Antarctic Facilities

Roberto Gilmozzi, European Southern Observatory (Germany)

20 Telescope Structures

Larry M. Stepp, Thirty Meter Telescope Project (United States) 
Downloaded From: https://www.spiedigitallibrary.org/conference-proceedings-of-spie on 26 Apr 2023

Terms of Use: https://www.spiedigitallibrary.org/terms-of-use 


\section{Introduction}

The 2008 Ground-based and Airborne Telescopes conference brought together a large number of participants from around the world, and their oral and poster papers are contained in these proceedings. In this year's conference we had more invited papers than usual (16), reflecting the large number of significant telescope projects currently active, and the results were favorable-the invited talks were very well attended and generated a lot of interest.

The conference offered outstanding geographical and technical diversity, with reports about astronomy projects on all seven continents, involving both groundbased and airborne telescopes working in the UV, visible, IR, and radio wavelengths, and engaged in night-time and solar astronomy. It covered a full range of activities including planning, designing, constructing, integrating, commissioning, and upgrading new and existing telescopes, from portable telescopes used for site testing to extremely large telescopes with collecting areas up to a square kilometer.

The conference sessions included project reports on ground-based optical-IR telescopes, radio and sub-mm telescopes, airborne telescopes, solar telescopes, Antarctic telescopes, and future giant telescopes. There were also sessions on a number of technical subjects, including site testing, integration, and commissioning; telescope alignment and control; telescope structures; wind loading, and segmented mirror alignment and phasing.

It is gratifying to see that the high level of telescope-building activity that developed in the 1990s has continued strong into the 21 st century, with successful completion of many projects and the initiation of many more that offer exciting scientific prospects for the coming decade. As many of the projects get bigger, finding funding for them will be a continuing challenge, but we are encouraged to see the emergence of strong astronomy programs in many of the rapidly developing countries of the world that offer the promise of broadening the base of astronomical facilities to serve this expanding community.

The conference chairs would like to thank the authors and conference attendees, the program committee members who devoted many hours to planning the conference and who served as the session chairs, and the conference organizers at SPIE.

Larry M. Stepp

Roberto Gilmozzi 
Downloaded From: https://www.spiedigitallibrary.org/conference-proceedings-of-spie on 26 Apr 2023

Terms of Use: https://www.spiedigitallibrary.org/terms-of-use 